\title{
Program to Improve Colorectal Cancer Screening in a Low-Income, Racially Diverse Population: A Randomized Controlled Trial
}

Muriel Jean-Jacques, $M D, M A^{1}$

Erin O. Kaleba, $\mathrm{MPH}^{2}$

Jobn L. Gatta, $P b D^{3}$

Gabriela Gracia, $M S^{4}$

Elizabeth R. Ryan, EdD ${ }^{3}$

Bechara N. Choucair, $M D^{3,5}$

'Division of General Internal Medicine, Department of Medicine, Feinberg School of Medicine, Northwestern University, Chicago, Illinois

${ }^{2}$ Alliance of Chicago Community Health Services, Chicago, Illinois

${ }^{3}$ Department of Family and Community Medicine, Northwestern University,

Chicago, Illinois

${ }^{4}$ Health Research and Educational Trust, Chicago, Illinois

${ }^{5}$ Chicago Department of Public Health, Chicago, Illinois

\begin{abstract}
PURPOSE More effective strategies are needed to improve rates of colorectal cancer screening, particularly among the poor, racial and ethnic minorities, and individuals with limited English proficiency. We examined whether the direct mailing of fecal occult blood testing (FOBT) kits to patients overdue for such screening is an effective way to improve screening in this population.
\end{abstract}

METHODS All adults aged 50 to 80 years who did not have documentation of being up to date with colorectal cancer screening as of December 31, 2009, and who had had at least 2 visits to the community health center in the prior 18 months were randomized to the outreach intervention or usual care. Patients in the outreach group were mailed a colorectal cancer fact sheet and FOBT kit. Patients in the usual care group could be referred for screening during usual clinician visits. The primary outcome was completion of colorectal cancer screening (by FOBT, sigmoidoscopy, or colonoscopy) 4 months after initiation of the outreach protocol. Outcome measures were compared using the Fisher exact test.

RESULTS Analyses were based on 104 patients assigned to the outreach intervention and 98 patients assigned to usual care. In all, $30 \%$ of patients in the outreach group completed colorectal cancer screening during the study period, compared with $5 \%$ of patients in the usual care group $(P<.001)$. Nearly all of the screenings were by FOBT. The groups did not differ significantly with respect to the percentage of patients making a clinician visit or the percentage for whom a clinician placed an order for a screening test.

CONCLUSIONS The mailing of FOBT kits directly to patients was efficacious for promoting colorectal cancer screening among a population with high levels of poverty, limited English proficiency, and racial and ethnic diversity. Non-visit-based outreach to patients may be an important strategy to address suboptimal rates of colorectal cancer screening among populations most at risk for not being screened.

Ann Fam Med 2012;10:412-417. doi:10.1370/afm.1381.

\section{INTRODUCTION}

olorectal cancer is the second leading cause of cancer death in the United States and the third most commonly diagnosed cancer. ${ }^{1}$ Appropriate screening and early detection can greatly reduce

Conflicts of interest: authors report none.

\section{CORRESPONDING AUTHOR}

Muriel Jean-Jacques, MD, MA 750 N Lake Shore Dr, 10th Floor Feinberg School of Medicine Northwestern University

Chicago, IL 60611

mjeanjacques@northwestern.edu colorectal cancer-associated morbidity and mortality, and several national guidelines recommend regular screening for colorectal cancer among adults aged 50 years and older with high-sensitivity fecal occult blood testing (FOBT), sigmoidoscopy, or colonoscopy. ${ }^{2-4}$ Nearly one-half of eligible adults are not up to date on colorectal cancer screening, however. ${ }^{5}$ Moreover, despite overall improvements in the rate of colorectal cancer screening, marked disparities persist, with lower rates of colorectal cancer screening among racial and ethnic minorities, individuals with lower income or lower educational attainment, the uninsured, and individuals 
who were not born in the United States. ${ }^{5.7}$ Rates of screening are particularly low for foreign-born individuals who immigrated to the United States more recently or are less acculturated..$^{6-8}$ There is thus a clear need to identify effective strategies to improve the use of colorectal cancer screening among medically underserved populations, and particularly among immigrants and individuals with limited English proficiency.

The direct mailing of FOBT kits to patients who are due for colorectal cancer screening has been shown to be both clinically effective $e^{9-14}$ and cost-effective $e^{15,16}$ for increasing colorectal cancer screening rates. The vast majority of studies assessing the efficacy of this strategy have not included sizable numbers of patients with lower socioeconomic status or from racial or ethnic minority groups, however. ${ }^{9-11,13,14}$ We therefore set out to examine the efficacy of an outreach intervention that includes the direct mailing of FOBT kits to patients who were overdue for colorectal cancer screening and were receiving care at a community health center that serves a primarily low income population with a high proportion of immigrants and refugees.

\section{METHODS}

\section{Study Setting}

This study was conducted at Heartland International Health Center (HIHC), a federally qualified health center that provides comprehensive primary care to a predominantly low income, uninsured, and racially diverse population on the north side of Chicago, Illinois. HIHC is an affiliate of Heartland Alliance, a nonprofit organization that provides housing, health care, economic, and legal services to immigrants, refugees, and the poor. HIHC serves more than 13,000 patients annually across 8 sites. Thirty percent of center patients are non-English speaking, and translation services are provided for more than 35 languages. This study was limited to a single HIHC site that serves more than 3,000 patients a year and is staffed by $3 \mathrm{fam}$ ily medicine physicians and 1 nurse practitioner. Of the patients who sought care at the study site in 2010, $48 \%$ were Hispanic, 24\% were black, and 6\% were Asian. Sixty-one percent of patients were uninsured, and 33\% had Medicaid. This site serves a large number of immigrants and refugees from Mexico, South America, SubSaharan Africa, Southeast Asia, and Eastern Europe.

HIHC has been using a commercially available electronic health record (EHR) system since 2006 and has regularly tracked its colorectal cancer screening rate in accordance with the Health Resources and Services Administration core measurement set guidelines. The Administration's colorectal cancer screening measure assesses the percentage of adults aged 50 to 80 years seen in the measurement year who have had an FOBT within 1 year, flexible sigmoidoscopy or doublecontrast barium enema within 5 years, or colonoscopy within 10 years. ${ }^{17}$ The percentage of patients across all HIHC sites who satisfied this measure was $17 \%$ in 2008 and $36 \%$ in 2009 .

\section{Eligibility Criteria}

Patients were eligible for this study if they were adults aged 50 to 80 years who had had at least 2 visits to the study site between July 1, 2008, and December 31, 2009 , with no history of colorectal cancer or total colectomy, and with no documented FOBT within 1 year, sigmoidoscopy within 5 years, or colonoscopy within 10 years as of December 31, 2009.

\section{Intervention}

The study was approved by the institutional review boards of Northwestern University and the Heartland Alliance, and a waiver of informed consent was obtained. All eligible patients were randomly assigned to usual care or the outreach intervention using a uniform random number generator. Patients assigned to usual care could be referred for colorectal cancer screening, per usual health center protocol, at clinician visits. Patients assigned to the outreach intervention were sent a mailing that included (1) a letter from their medical professional notifying them that it was time for their colorectal cancer screening examination and encouraging them to be screened through FOBT, (2) a colorectal cancer fact sheet from the Centers for Disease Control and Prevention in both English and Spanish, ${ }^{18,19}$ (3) a Hemoccult II SENSA Elite kit (Beckman Coulter, Inc), which is a 3 -sample guaiac-based FOBT test, and (4) manufacturer-supplied instructions regarding proper patient preparation, sample collection, and FOBT kit handling. Patients were instructed to return the completed FOBT kit to the health center laboratory in person or via postage-paid envelope.

Patients who did not return an FOBT kit within 2 weeks of the mailing received telephone outreach by a lay health educator who is bilingual in English and Spanish. The outreach included up to 3 telephone call attempts each spaced 2 weeks apart. A script was used to guide the calls; during each call, the outreach coordinator confirmed whether the patient had received the mailing, inquired about and addressed any questions the patient may have had regarding colorectal cancer screening in general and FOBT specifically, and reminded the patient to complete and return the FOBT kit. Patients who were not reached within 3 call attempts and who did not return the FOBT kit within 6 weeks of the initial mailing were sent a second mailing that included a letter from their medical professional 
encouraging them to be screened for colorectal cancer and another FOBT kit.

One of the authors (M.J-J.) trained and supervised the outreach coordinator. All print materials were provided in English and Spanish, and the outreach coordinator used a telephone-based interpretation service to communicate with patients who were not proficient in English or Spanish. FOBT cards were processed by the health center laboratory staff according to usual protocol. Patients were informed of their FOBT results by their primary care clinician by letter or telephone according to usual clinic practice, and patients with positive FOBT results were referred for colonoscopy. The outreach was conducted from February 25, 2010, through April 30, 2010.

\section{Outcome Measures}

The main outcome measure was the percentage of eligible patients who completed a colorectal cancer screening test (FOBT, sigmoidoscopy, or colonoscopy) by June 30 , 2010,4 months after initiation of the outreach protocol. Secondary outcome measures included the percentage of patients who had a clinician visit during the study period, the percentage of eligible patients whose clinician placed an order for FOBT during the study period, and the type of colorectal cancer screening test performed. Patient eligibility, patients' demographic information, and study outcomes were assessed by querying the EHR data warehouse. Patient race/ethnicity and language preference were self-reported the time of registration and recorded in the EHR by registration staff.

\section{Statistical Analysis}

We compared the proportion of patients achieving each of the outcome measures in the control and intervention groups using the Fisher exact test. Demographic characteristics of patients randomized to the usual care and intervention groups were compared using the Pearson $\chi^{2}$ test or Student $t$ test as appropriate. We used a 2 -sided $P$ value of less than .05 as the threshold for statistical significance. Statistical analyses were conducted using SPSS version 19 (IBM Corporation).

\section{RESULTS}

\section{Demographics}

A total of 317 patients aged 50 to 80 years had at least 2 visits to the study site between July 1, 2008, and December 31, 2009, and were eligible for the colorectal cancer screening measure. Of these, 202 (64\%) did not have documented evidence of an appropriate colorectal cancer screening test as of December 31, 2009, and were therefore eligible for the study. In all, 98 patients were randomized to the usual care group and 104 were randomized to the outreach intervention. The patients were racially and ethnically diverse, more than one-half of patients were women, and the vast majority were uninsured or publicly insured (Table 1).

\section{Outcomes}

In terms of the main outcome, $30 \%$ of patients (31 of 104) assigned to the outreach intervention and 5\% of patients ( 5 of 98) assigned to the usual care group completed colorectal cancer screening between January 1, 2010, and June 30, $2010(P<.001)$ (Table 2).

Almost all of the completed colorectal cancer screening tests were FOBTs.

In addition, $44 \%$ of patients (46 of 104) assigned to the outreach intervention and $49 \%$ of patients ( 48

\begin{tabular}{|c|c|c|c|}
\hline Characteristic & $\begin{array}{l}\text { Usual Care } \\
(n=98)\end{array}$ & $\begin{array}{l}\text { Intervention } \\
(n=104)\end{array}$ & $\begin{array}{c}P \\
\text { Value }\end{array}$ \\
\hline Female, No. (\%) & $58(59)$ & $67(64)$ & .44 \\
\hline Age, mean $\pm S D, y$ & $60 \pm 8$ & $60 \pm 7$ & .69 \\
\hline Race/ethnicity, No. (\%) & & & .65 \\
\hline White & $23(24)$ & $30(29)$ & \\
\hline Black & $27(28)$ & $28(27)$ & \\
\hline Hispanic & $21(21)$ & $20(19)$ & \\
\hline Asian & $12(12)$ & $16(15)$ & \\
\hline Multiracial & $1(1)$ & $2(2)$ & \\
\hline Other/unknown & $14(14)$ & $8(8)$ & \\
\hline $\begin{array}{l}\text { Health insurance, } \\
\text { No. (\%) }\end{array}$ & & & .08 \\
\hline Uninsured & $73(74)$ & $64(62)$ & \\
\hline Medicaid & $5(5)$ & $16(15)$ & \\
\hline Medicare & $16(16)$ & $18(17)$ & \\
\hline Other & $4(4)$ & $6(6)$ & \\
\hline $\begin{array}{l}\text { Language preference, } \\
\text { No. (\%) }\end{array}$ & & & .80 \\
\hline English & $36(37)$ & $42(40)$ & \\
\hline Spanish & $20(20)$ & $18(17)$ & \\
\hline Other & $42(43)$ & $44(42)$ & \\
\hline
\end{tabular}

Table 2. Patients Completing Colorectal Cancer Screening Tests by June 30, 2010, by Group

\begin{tabular}{lccc}
\hline Screening Test & $\begin{array}{c}\text { Usual Care } \\
(\mathbf{n}=\mathbf{9 8})\end{array}$ & $\begin{array}{c}\text { Intervention } \\
(\mathbf{n}=\mathbf{1 0 4})\end{array}$ & $\begin{array}{c}P \\
\text { Value }\end{array}$ \\
\hline Any, ${ }^{\text {a No. }(\%)}$ & $5(5)$ & $31(30)$ & $<.001$ \\
Type of test & 4 & 30 & - \\
FOBT, No. & 1 & 1 & - \\
Colonoscopy, No. & 0 & 0 & - \\
Sigmoidoscopy, No. & \multicolumn{4}{l}{} \\
\hline FOBT = fecal occult blood test. & & \\
any includes FOBT, colonoscopy, or sigmoidoscopy. &
\end{tabular}


of 98) assigned to the control group made a clinician visit during this time frame, a difference that was not statistically significant $(P=.57)$. Also, $4 \%$ (4 of 104) of patients assigned to the outreach intervention group and 5\% (5 of 98) of patients assigned to the control group had an order for colorectal cancer screening placed by their clinician $(P=.74)$.

Process Assessment for the Intervention Group One FOBT test was returned within 2 weeks of the initial mailing. The outreach coordinator attempted to call the remaining 103 patients assigned to the intervention group. Forty-one of the 103 patients (40\%) were reached within 3 telephone call attempts: 25 on the first call, 12 on the second call, and 4 on the third call. Twenty-four of the 103 patients (23\%) had telephone numbers that were wrong or not in service. Fifteen of the 30 completed FOBT kits were returned within 6 weeks of the initial mailing. A second FOBT kit was mailed to the 57 patients ( $55 \%$ of those randomized to the outreach group) who had not been reached within 3 call attempts and who had not returned an FOBT kit within 6 weeks of the initial mailing.

\section{Post Hoc Analysis}

The prespecified primary study outcome was completion of FOBT, sigmoidoscopy, or colonoscopy by June 30,2010 ; however, because of concern that this short follow-up period may have biased results in favor of the intervention group (as many patients in the usual care group may not have had a clinic visit and opportunity to be referred for colorectal cancer screening in this time interval), we also examined completion of a colorectal cancer screening test by March 24, 2011, 12 months after the last batch of the initial outreach mailings were sent. Including patients who had completed a screening test by June 30, 2010, 40 of 104 patients (38\%) from the intervention group and 15 of 98 patients (15\%) from the usual care group completed a colorectal cancer screening test by March 24, $2011(P=.002)$.

\section{DISCUSSION}

In this randomized controlled trial, an outreach intervention that consisted of the mailing of FOBT kits to patients overdue for colorectal cancer screening with additional telephone outreach to initial nonresponders was efficacious for promoting colorectal cancer screening among a population with high levels of poverty, lack of health insurance, limited English proficiency, racial and ethnic diversity, and other features associated with a lower likelihood of receiving colorectal cancer screening. Although prior studies have shown that the direct-to-patient mailing of FOBT kits can lead to higher colorectal cancer screening rates in predominantly white, middle-class, or well-insured populations, ${ }_{1}^{9-11,13,14}$ few studies have assessed the efficacy of this strategy in historically underserved populations. Those that have evaluated this intervention in more vulnerable populations have tended to focus on just 1 or 2 ethnic groups. ${ }^{12,20}$ Our study adds to this existing literature by demonstrating that this outreach strategy can significantly improve colorectal cancer screening rates even among economically disadvantaged patients from a wide range of racial, ethnic, and cultural backgrounds.

Especially in settings with an EHR system to facilitate the ready identification of patients who are eligible and overdue for colorectal cancer screening, the direct mailing of FOBT kits to eligible patients with some additional phone outreach to initial nonresponders may be a sustainable strategy to promote colorectal cancer screening, particularly under a patient-centered medical home payment model that reimburses practices for population health management activities. ${ }^{21}$ Although our study did not include formal cost or cost-effectiveness analyses, very similar outreach interventions have been found to be cost-effective in other studies. ${ }^{15,16}$

Our study demonstrated that the direct-to-patient outreach intervention was efficacious even in a health care setting that had already implemented point-ofcare clinician-directed electronic clinical reminders to promote appropriate colorectal cancer screening. This finding is in keeping with prior studies that have shown the superiority of patient-directed outreach over clinician-directed reminders ${ }^{13}$ and the limited efficacy of clinician-directed reminders on colorectal cancer screening rates. ${ }^{22-25}$ Although the reasons for the low and in some cases lack of efficacy of cliniciandirected reminders on colorectal cancer screening are not completely clear, the evidence overall suggests that patient-directed reminders may be a crucial component of efforts to improve colorectal cancer screening rates.

The use of patient-directed outreach to promote colorectal cancer screening, particularly through FOBT, may also be an important strategy to address disparities in colorectal cancer screening. Although the US Preventive Services Task Force gives equal recommendation to colorectal cancer screening with FOBT, sigmoidoscopy, or colonoscopy, ${ }^{2}$ some professional societies recommend colonoscopy as the preferred method of screening, ${ }^{4}$ and some physicians focus heavily on colonoscopy when discussing colorectal cancer screening with patients ${ }^{26}$; however, because of the ongoing financial barriers to colonoscopy, particularly among the uninsured the limited access to colonoscopy in some areas, even among the insured; and the preference of some patients for FOBT over other methods of colorectal cancer screening, ${ }^{27}$ the use of patient-directed outreach that includes 
FOBT may be an effective way to promote colorectal cancer screening, especially among those who are least likely to complete endoscopic screening tests.

It is important to note that despite outreach efforts, $70 \%$ of patients assigned to the intervention arm of our study did not complete a colorectal cancer screening test during the study period. The use of simplified testing instructions ${ }_{1}^{12}$ culturally tailored screening promotion materials, ${ }^{28,29}$ or single-sample fecal immunochemical tests ${ }^{30}$ could be incorporated into future iterations of this intervention to address possible literacy, cultural, and logistic barriers to fecal-based colorectal cancer screening, and could potentially improve the efficacy of the intervention. Other strategies, including the use of patient navigators, ${ }^{31-33}$ peer educators, ${ }^{34}$ decision aides, ${ }^{35,36}$ or community-based outreach efforts, ${ }^{37-39}$ may be particularly appropriate for patients who have not completed colorectal cancer screening despite mail and telephone-based outreach efforts. Furthermore, the efficacy of FOBT screening is considered to be equivalent to endoscopic screening only if positive FOBT tests are appropriately followed up and if the adherence to repeated FOBT screening is high. ${ }^{40}$ Further efforts will therefore be needed to ensure appropriate endoscopic follow-up of positive FOBT tests and adequate adherence, not only to 1-time, but also to repeated FOBT screening.

Our study has several limitations. First, as a small, single-center study in a historically underserved population, our results may not be generalizable; however, our findings address the potential lack of generalizability of prior studies of similar outreach strategies to vulnerable populations. Second, the use of automated queries of the EHR data warehouse to determine patient eligibility and assess outcomes, although highly standardized, is imperfect. We did not have access to colorectal cancer screening tests that were done outside of Heartland International Health Outreach, and some tests and procedures that were completed before 2006 and scanned into the EHR at the time of the 2006 EHR conversion may not have been appropriately captured. In addition, some patients classified as eligible for colorectal cancer screening by age criteria alone may have been inappropriate candidates for screening because of comorbid conditions or limited life expectancy. These potential sources of error should have affected the intervention and control groups equally given the randomized study design, however, and thus should not have affected the main study findings.

Another important limitation of our study is related to the timing of our primary outcome assessment. The prespecified 4-month follow-up period could have biased the results in favor of the outreach group; however, a post hoc analysis at 12 months of follow-up still showed a significant difference in screening completion between groups favoring the outreach group.

In conclusion, this randomized controlled study demonstrated that a cost-effective outreach intervention consisting of the direct mailing of FOBT kits to patients overdue for colorectal cancer screening and telephone outreach to initial nonresponders was an effective means to increase patient completion of colorectal cancer screening, even in a population with a high proportion of patients from racial, ethnic, socioeconomic, and English-proficiency groups that are most at risk for not receiving appropriate colorectal cancer screening. As this outreach strategy overcomes several financial barriers (eg, sliding-scale fee for preventive visit, missed wages due to time off from work) and nonfinancial barriers (eg, transportation challenges, time away from family obligations) associated with visit-initiated cancer screening, the direct mailing of FOBT kits to patients eligible for colorectal cancer screening may be a particularly desirable approach to improving adherence to colorectal cancer screening guidelines in historically underserved communities.

To read or post commentaries in response to this article, see it online at http://www.annfammed.org/content/10/5/412.

Key words: fecal occult blood testing; cancer, colorectal; cancer screening; special populations, underserved/minority; health promotion/disease prevention, screening; practice-based research

Submitted July 21, 2011; submitted, revised, October 19, 2011; accepted December 1, 2011.

Funding support: This study was funded through a seed grant from the Northwestern University Clinical and Translational Sciences Institute (NUCATS). Dr Jean-Jacques was supported in part by federal funds from the National Center for Research Resources (NCRR) and National Institutes of Health (NIH) through the Clinical and Translational Science Awards Program (CTSA) (Northwestern University UL1RR0254741).

Acknowledgments: We would like to thank Susan Hossli from Heartland International Health Center for her assistance in facilitating and coordinating the provision of FOBT kits. We would also like to thank Brendan Stubbs, Nathan Taylor, and Eduardo Segovia from the Alliance of Chicago Community Health Services for their work conducting the electronic health record system data warehouse queries. We are indebted to the clinicians, staff, and patients of Heartland International Health Center who graciously participated in focus groups that aided in the design of the outreach intervention.

\section{References}

1. American Cancer Society. Cancer Facts \& Figures 2010. Atlanta, GA: American Cancer Society; 2010. http://www.cancer.org/Research/ CancerFactsFigures/CancerFactsFigures/cancer-facts-and-figures-2010. Accessed Aug 22, 2012.

2. US Preventive Services Task Force. Screening for Colorectal Cancer: U.S. Preventive Services Task Force Recommendation Statement. AHRQ Publication 08-05124-EF-3, October 2008. http://www. uspreventiveservicestaskforce.org/uspstf08/colocancer/colors.htm. Accessed Aug 22, 2012. 
3. Levin B, Lieberman DA, McFarland B, et al; American Cancer Society Colorectal Cancer Advisory Group; US Multi-Society Task Force; American College of Radiology Colon Cancer Committee. Screening and surveillance for the early detection of colorectal cancer and adenomatous polyps, 2008: a joint guideline from the American Cancer Society, the US Multi-Society Task Force on Colorectal Cancer, and the American College of Radiology. Gastroenterology. 2008;134(5):1570-1595.

4. Rex DK, Johnson DA, Anderson JC, Schoenfeld PS, Burke CA, Inadomi JM; American College of Gastroenterology. American College of Gastroenterology guidelines for colorectal cancer screening 2009 [corrected]. Am J Gastroenterol. 2009;104(3):739-750.

5. National Center for Health Statistics. Health, United States, 2010: With Special Feature on Death and Dying. Hyattsville, MD: National Center for Health Statistics; 2011:18.

6. Shih YC, Elting LS, Levin B. Disparities in colorectal screening between US-born and foreign-born populations: evidence from the 2000 National Health Interview Survey. J Cancer Educ. 2008;23(1):18-25.

7. Holden DJ, Jonas DE, Porterfield DS, Reuland D, Harris R. Systematic review: enhancing the use and quality of colorectal cancer screening. Ann Intern Med. 2010;152(10):668-676.

8. Johnson-Kozlow M. Colorectal cancer screening of Californian adults of Mexican origin as a function of acculturation. J Immigr Minor Health. 2010;12(4):454-461.

9. Ore L, Hagoel L, Lavi I, Rennert G. Screening with faecal occult blood test (FOBT) for colorectal cancer: assessment of two methods that attempt to improve compliance. Eur J Cancer Prev. 2001;10(3):251-256.

10. Church TR, Yeazel MW, Jones RM, et al. A randomized trial of direct mailing of fecal occult blood tests to increase colorectal cancer screening. J Natl Cancer Inst. 2004;96(10):770-780.

11. Myers RE, Sifri R, Hyslop T, et al. A randomized controlled trial of the impact of targeted and tailored interventions on colorectal cancer screening. Cancer. 2007;110(9):2083-2091.

12. Coronado GD, Golovaty I, Longton G, Levy L, Jimenez R. Effectiveness of a clinic-based colorectal cancer screening promotion program for underserved Hispanics. Cancer. 2011;117(8):1745-1754.

13. Sequist TD, Zaslavsky AM, Marshall R, Fletcher RH, Ayanian JZ. Patient and physician reminders to promote colorectal cancer screening: a randomized controlled trial. Arch Intern Med. 2009; $169(4): 364-371$

14. Hoffman RM, Steel SR, Yee EF, et al. A system-based intervention to improve colorectal cancer screening uptake. Am J Manag Care. 2011;17(1):49-55.

15. Lairson DR, DiCarlo M, Myers RE, et al. Cost-effectiveness of targeted and tailored interventions on colorectal cancer screening use. Cancer. 2008;112(4):779-788.

16. Sequist TD, Franz C, Ayanian JZ. Cost-effectiveness of patient mailings to promote colorectal cancer screening. Med Care. 2010;48(6): 553-557.

17. Health Resources and Services Administration. US Department of Health and Human Services. HRSA 12 Core Clinical Measures. Rockville, MD: HRSA. http://www.hrsa.gov/healthit/coremeasures.html. Accessed Aug 22, 2012

18. Centers for Disease Control and Prevention. Colorectal Cancer Screening Basic Fact Sheet. Rockville, MD: CDC, 2009. Publication 99-6949.

19. Centers for Disease Control and Prevention. Pruebas de Deteccion de Cancer Colorrectal Informacion Basica. Publication 99-7515. Rockville, MD: CDC; 2009.

20. Walsh JM, Salazar R, Kaplan C, Nguyen L, Hwang J, Pasick RJ. Healthy Colon, Healthy Life (Colon Sano, Vida Sana): colorectal cancer screening among Latinos in Santa Clara, California. J Cancer Educ. 2010;25(1):36-42.

21. American Academy of Family Physicians (AAFP), American Academy of Pediatrics (AAP), American College of Physicians (ACP), American Osteopathic Association (AOA). Joint Principles of the Patient-Centered Medical Home. February 2007. http://www.pcpcc.net/content/jointprinciples-patient-centered-medical-home. Accessed Aug 22, 2012.
22. Dietrich AJ, Tobin JN, Sox $\mathrm{CH}$, et al. Cancer early-detection services in community health centers for the underserved. A randomized controlled trial. Arch Fam Med. 1998;7(4):320-327, discussion 328.

23. Ganz PA, Farmer MM, Belman MJ, et al. Results of a randomized controlled trial to increase colorectal cancer screening in a managed care health plan. Cancer. 2005;104(10):2072-2083.

24. Roetzheim RG, Christman LK, Jacobsen PB, et al. A randomized controlled trial to increase cancer screening among attendees of community health centers. Ann Fam Med. 2004;2(4):294-300.

25. Roetzheim RG, Christman LK, Jacobsen PB, Schroeder J, Abdulla R, Hunter $\mathrm{S}$. Long-term results from a randomized controlled trial to increase cancer screening among attendees of community health centers. Ann Fam Med. 2005:3(2):109-114.

26. McQueen A, Bartholomew LK, Greisinger AJ, et al. Behind closed doors: physician-patient discussions about colorectal cancer screening. J Gen Intern Med. 2009;24(11):1228-1235.

27. El-Nachef N, Thomas JP, Lin YV, et al. Racial differences in barriers to colorectal cancer screening. Gastroenterology. 2010;138(1 Suppl 5):S189.

28. Walsh JM, Salazar R, Nguyen TT, et al. Healthy colon, healthy life: a novel colorectal cancer screening intervention. Am J Prev Med. 2010;39(1):1-14

29. Aragones A, Schwartz MD, Shah NR, Gany FM. A randomized controlled trial of a multilevel intervention to increase colorectal cancer screening among Latino immigrants in a primary care facility. J Gen Intern Med. 2010;25(6):564-567.

30. Daly JM, Levy BT, Merchant ML, Wilbur J. Mailed fecal-immunochemical test for colon cancer screening. J Community Health. 2010; 35(3):235-239.

31. Jandorf L, Gutierrez Y, Lopez J, Christie J, Itzkowitz SH. Use of a patient navigator to increase colorectal cancer screening in an urban neighborhood health clinic. J Urban Health. 2005;82(2):216-224.

32. Percac-Lima S, Grant RW, Green AR, et al. A culturally tailored navigator program for colorectal cancer screening in a community health center: a randomized, controlled trial. J Gen Intern Med. 2009;24(2):211-217.

33. Christie J, Itzkowitz S, Lihau-Nkanza I, Castillo A, Redd W, Jandorf $\mathrm{L}$. A randomized controlled trial using patient navigation to increase colonoscopy screening among low-income minorities. J Natl Med Assoc. 2008;100(3):278-284.

34. Lasser KE, Murillo J, Lisboa S, et al. Colorectal cancer screening among ethnically diverse, low-income patients: a randomized controlled trial. Arch Intern Med. 2011;171(10):906-912

35. Miller DP Jr, Spangler JG, Case LD, Goff DC Jr, Singh S, Pignone MP. Effectiveness of a web-based colorectal cancer screening patient decision aid: a randomized controlled trial in a mixedliteracy population. Am J Prev Med. 2011;40(6):608-615

36. Ruffin MT 4th, Fetters MD, Jimbo M. Preference-based electronic decision aid to promote colorectal cancer screening: results of a randomized controlled trial. Prev Med. 2007;45(4):267-273.

37. Ma GX, Shive S, Tan Y, et al. Community-based colorectal cancer intervention in underserved Korean Americans. Cancer Epidemiol. 2009;33(5):381-386.

38. Maxwell AE, Bastani R, Danao LL, Antonio C, Garcia GM, Crespi CM. Results of a community-based randomized trial to increase colorectal cancer screening among Filipino Americans. Am J Public Health. 2010;100(11):2228-2234.

39. Blumenthal DS, Smith SA, Majett CD, Alema-Mensah E. A trial of 3 interventions to promote colorectal cancer screening in African Americans. Cancer. 2010;116(4):922-929.

40. Zauber AG, Lansdorp-Vogelaar I, Knudsen AB, Wilschut J, van Ballegooijen M, Kuntz KM. Evaluating test strategies for colorectal cancer screening: a decision analysis for the U.S. Preventive Services Task Force. Ann Intern Med. 2008;149(9):659-669. 